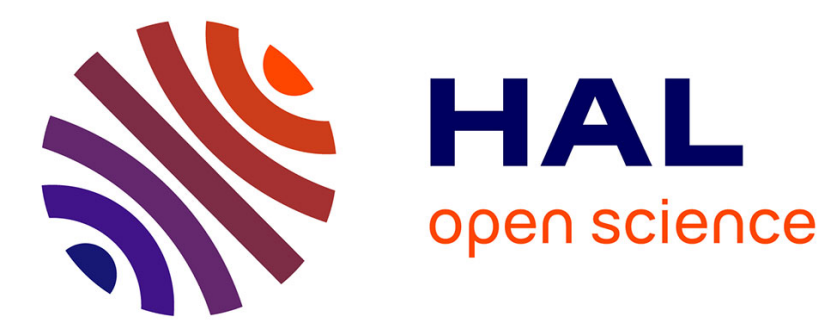

\title{
Homogenization estimates for texture evolution in halite
} Yi Liu, Pierre Gilormini, Pedro Ponte Castañeda

\section{To cite this version:}

Yi Liu, Pierre Gilormini, Pedro Ponte Castañeda. Homogenization estimates for texture evolution in halite. Tectonophysics, 2005, 406, pp.179-195. 10.1016/j.tecto.2005.06.007 . hal-00111503

\section{HAL Id: hal-00111503 https://hal.science/hal-00111503}

Submitted on 14 Aug 2019

HAL is a multi-disciplinary open access archive for the deposit and dissemination of scientific research documents, whether they are published or not. The documents may come from teaching and research institutions in France or abroad, or from public or private research centers.
L'archive ouverte pluridisciplinaire HAL, est destinée au dépôt et à la diffusion de documents scientifiques de niveau recherche, publiés ou non, émanant des établissements d'enseignement et de recherche français ou étrangers, des laboratoires publics ou privés. 


\title{
Homogenization estimates for texture evolution in halite
}

\author{
Yi Liu ${ }^{a}$, Pierre Gilormini ${ }^{\mathrm{b}}$, Pedro Ponte Castañeda ${ }^{\mathrm{a}, \mathrm{c}, *}$ \\ ${ }^{a}$ Department of Mechanical Engineering and Applied Mechanics, University of Pennsylvania, Philadelphia, PA 19104-6315, U.S.A \\ ${ }^{\mathrm{b}}$ Laboratoire d'Ingénierie des Matériaux (CNRS UMR 8006), ENSAM, 151 Boulevard de l'Hôpital, 75013 Paris, France \\ ${ }^{\mathrm{c}}$ Laboratoire de Mécanique des Solides (CNRS UMR 7649), Département de Mécanique, Ecole Polytechnique, 91128 Palaiseau, France
}

\begin{abstract}
In this work, the recently developed bsecond-orderQ self-consistent method [Liu, Y., Ponte Castañeda, P., 2004a. Secondorder estimates for the effective behavior and field fluctuations in viscoplastic polycrystals. J. Mech. Phys. Solids 52 467-495] is used to simulate texture evolution in halite polycrystals. This method makes use of a suitably optimized linear comparison polycrystal and has the distinguishing property of being exact to second order in the heterogeneity contrast. The second-order model takes into consideration the effects of hardening and of the evolution of both crystallographic and morphological texture to yield reliable predictions for the macroscopic behavior of the polycrystal. Comparisons of these predictions with fullfield numerical simulations [Lebensohn, R.A., Dawson, P.R., Kern, H.M., Wenk, H.R., 2003. Heterogeneous deformation and texture development in halite polycrystals: comparison of different modeling approaches and experimental data. Tectonophysics 370 287-311], as well as with predictions resulting from the earlier bvariationalQ and btangentQ selfconsistent models, included here for comparison purposes, provide insight into how the underlying assumptions of the various models affect slip in the grains, and therefore the texture predictions in highly anisotropic and nonlinear polycrystalline materials. The bsecond-orderQ self-consistent method, while giving a softer stress-strain response than the corresponding fullfield results, predicts a pattern of texture evolution that is not captured by the other homogenization models and that agrees reasonably well with the full-field predictions and with the experimental measures.
\end{abstract}

Keywords: Halite deformation; Creep; Polycrystal plasticity; Texture evolution; Variational methods; Self-consistent model

\footnotetext{
* Corresponding author. Laboratoire de Mécanique des Solides (CNRS UMR 7649), Département de Mécanique, Ecole Polytechnique, 91128 Palaiseau, France.

E-mail addresses: yiliu2@seas.upenn.edu (Y. Liu), pierre.gilormini@paris.ensam.fr (P. Gilormini), ponte@1ms.polytechnique.fr, ponte@seas.upenn.edu (P. Ponte Castañeda).
}

\section{Introduction}

Salt domes have been recognized as potential storage sites for nuclear waste since the middle of last century (e.g., Carter and Hansen, 1983; Hunsche and Hampel, 1999; Peach and Spiers, 1996; Aubertin and Hardy, 1998). This fact has motivated numerous studies for halite both for single crystals (Carter and 
Heard, 1970; Guillopé and Poirier, 1979) and polycrystals (Heard, 1972; Wawersik and Zeuch, 1986; Carter et al., 1993; Franssen, 1994; Wenk, 1999; Ter Heege et al., in press). Evidence from single-crystal experiments suggests that halite crystals exhibit strong degrees of nonlinearity and anisotropy at room temperature. Since the deformation mechanism of the single-crystal grains is fairly well understood, the question arises as to how to average the response of the huge numbers of singlecrystal grains in a typical macroscopic sample (Kocks et al., 1998). This question becomes particularly difficult when the polycrystalline samples are subjected to deformations where the relevant mechanisms (mostly dislocation motion and interaction) become strongly nonlinear, at least as manifested at the continuum level. It is known that the effective or overall response of a macroscopic polycrystalline sample depends not only on the single-crystal properties, but also on the microstructure (e.g., crystallographic texture and average grain shape) and its evolution during the deformation process. The role of homogenization is to establish this relation between the single-crystal properties, the microstructure and its evolution, and the overall response.

The simplest and still most commonly used homogenization procedure in polycrystalline plasticity is the uniform strain-rate approximation of Taylor (1938). There is also the corresponding uniform-stress approximation of Reuss (1929). For viscoplastic polycrystals, these approximations are known to provide rigorous upper and lower bounds, respectively, for the effective flow stress of the polycrystal. Improved methods, based on various types of ad hoc approximations, include several extensions of the self-consistent model, such as the "incremental" method of Hill (1965) and Hutchinson (1976), and the "tangent" procedure of Molinari et al. (1987) and Lebensohn and Tomé (1993). While these various approximations generally provide improvements on the Taylor and Reuss bounds, and reduce to the linear self-consistent estimate, on which they are all based, for linearly viscous behavior, they give widely diverging predictions for low rate-sensitivity materials. In particular, in the rate-insensitive limit, some of these estimates tend to the Taylor upper bound, while others tend to the Reuss lower bound. More recently, Nebozhyn et al. (2001) proposed the use of the self-consistent approximation within the context of the "linear comparison method" of deBotton and Ponte Castañeda (1995) to generate "variational" self-consistent estimates for various types of polycrystals. These estimates were found to be much softer than the Taylor bound, especially at low strain-rate sensitivities and for large grain anisotropy, and have the additional advantage that they can be interpreted as upper bounds for all other nonlinear estimates based on the linear self-consistent approximation. This clarified some issues associated with the rate-insensitive limit and allowed the elimination of some of the selfconsistent schemes, such as the "incremental" and "secant" schemes, because they were found to violate the variational bound for self-consistent estimates. However, precisely because of their upper bound property, the variational self-consistent estimates are also expected to be overestimates for the effective behavior and it is of interest to develop more accurate estimates of the self-consistent type, which will necessarily have to be softer than the variational self-consistent estimates. This was accomplished very recently (Liu and Ponte Castañeda, 2004a,b) by means of the "second-order" method (Ponte Castañeda, 2002), which derives its name from the distinguishing feature that it leads to estimates that are exact to second-order in the heterogeneity contrast. In other words, these estimates have the unique property of recovering exact perturbation expansion estimates based on a small parameter measuring the difference of the properties in the different phases of a heterogeneous material, such as a polycrystal.

The Taylor model was first applied to simulate deformation textures in halite by Wenk et al. (1989) (also reported in Wenk, 1999). For polycrystals whose highly symmetric constituent crystals have plenty of slip systems to accommodate arbitrary deformation, such as face-centered cubic metals, the Taylor model yields fairly good estimates for both the macroscopic stress-strain relation and texture development (e.g., Kocks et al., 1998). However, because of the physically incorrect hypothesis of uniform strain-rate fields in the polycrystal, as the crystal behavior becomes highly nonlinear and/or anisotropic, the Taylor model predicts overly stiff material properties and in some cases leads to incorrect texture deformation patterns (Siemes, 1974). Recent studies on halite deformed at room temperature (Wenk et al., 1989; Lebensohn et al., 2003; Liu, 2003) have shown that the Taylor model 
predicts a pure [111] extension texture when an axially symmetric extension is applied, which is in disagreement with the corresponding experimental results where both [111] and [001] components are observed.

The tangent, or viscoplastic self-consistent, model was first introduced by Molinari et al. (1987) and further developed by Lebensohn and Tomé (1993, 1994) who used an anisotropic medium as the reference linear comparison material. For highly nonlinear and/or anisotropic polycrystals, it has been observed to significantly underestimate the effective flow stress (e.g., Lebensohn and Tomé, 1993; Nebozhyn et al., 2001). On the other hand, the more recent variational self-consistent model of deBotton and Ponte Castañeda (1995) has been shown to be robust for highly nonlinear and/or anisotropic cases (Nebozhyn et al., 2001) and yields promising predictions for the deformation textures in commercially pure titanium (Liu et al., 2003). However, it was found to lead to texture predictions that are very similar to those resulting from the Taylor model for halite at high temperatures (Liu et al., 2003). A further weakness of the Taylor, tangent and variational self-consistent models, that has also been observed in our previous simulations for halite and titanium, is the overly sharp texture they predict in some cases. For instance, an excessive [111] intensity has been predicted by these models that is not observed in the corresponding extrusion experiments for halite at high temperature (Liu, 2003). Recent studies show that the second-order self-consistent model (Ponte Castañeda, 2002; Liu and Ponte Castañeda, 2004a,b) is in excellent agreement with full-field numerical results for both the effective behavior and field quantities in polycrystals (Lebensohn et al., 2004a,b). In the latter references, the full-field simulations make use of fast Fourier transforms (Michel et al., 2000) instead of the finite element method, as used, for example, by Balasubramanian and Anand (2002). A full-field numerical simulation, known as the "hybrid element polycrystal" (HEP) approach (Beaudoin et al., 1995), has also been employed to model various polycrystalline aggregates, including metals (Barton and Dawson, 2001) and halite (Lebensohn et al., 2003). Based on a hybrid variational principle for the heterogeneous fields in each element, it allows more flexible deformation than the standard finite element formulation. Consequently, the HEP simulations are believed to be more accurate in simulating the deformation of poly- crystalline materials than other types of numerical simulations.

In the present work, the second-order self-consistent method for polycrystals is extended to account for microstructure evolution during finite deformation processes, and is applied to halite deformed at room temperature. Expressions for the macroscopic stressstrain relation of the polycrystal, the average slip rate, strain rate and spins in the constituent crystals are derived consistently with the second-order theory. The evolution equations for the texture variables are then derived, with use of standard kinematical arguments. An application is then carried out for extrusion of initially isotropic halite at room temperature $\left(20{ }^{\circ} \mathrm{C}\right)$, and comparisons are made with the experimental measurements reported by Lebensohn et al. (2003), and with the corresponding predictions of the Taylor, tangent and variational self-consistent models and of the HEP numerical approach. The initial instantaneous response of the material is first investigated. This is followed by a simulation of a $30 \%$ strain test in uniaxial tension, where both the macroscopic stress-strain relation and the final deformation texture are addressed.

\section{Theory}

\subsection{Second-order self-consistent estimates}

In this work, a polycrystal will be taken to be an aggregate of randomly orientated and distributed, perfectly bonded single-crystal grains with prescribed orientation distribution function (ODF) and associated two-point correlation function (see, for example, Adams and Olson, 1998). The ODF determines the distribution of the lattice orientation in crystals, i.e. the crystallographic texture, and the corresponding two-point statistics correlate with grain shape, so that the two-point correlation function serves to characterize (in an approximate way) the morphological texture, as determined by the average shape of the grain. For simplicity, it will be assumed here that the lattice orientations take on a set of discrete values, characterized by rotation tensors $\mathbf{Q}^{(r)}(r=1, \ldots, N)$. The polycrystal is assumed to occupy a region $\Omega$, while all the grains of a given orientation $\mathbf{Q}^{(r)}$ occupy subregion $\Omega^{(r)}(r=1, \ldots, N)$. The characteristic func- 
tions $\chi^{(r)}$ describing the location of the various orientations are equal to 1 if the position vector $\mathbf{x}$ is in $\Omega^{(r)}$, and zero otherwise. Volume averages over $\Omega$ are denoted by $\langle$.$\rangle , so that the scalars c^{(r)}=\left\langle\chi^{(r)}\right\rangle$ characterize the crystallographic texture of the polycrystal. The corresponding two-point correlation functions $p^{(r s)}=$ $\left\langle\chi^{(r)} \chi^{(s)}\right\rangle$ are assumed to exhibit "ellipsoidal" symmetry (Willis, 1977) with the same shape for all crystal orientations, characterized by a symmetric tensor $\mathbf{Z}$ (i.e., on the average, all grains are ellipsoidal in shape with identical aspect ratios and orientation).

For a given stress $\boldsymbol{\sigma}$ acting on a crystal with orientation $\mathbf{Q}^{(r)}$, the slip rate $\gamma_{(k)}^{(r)}$ on the $k$-th system $(k=1, \ldots, K)$ is assumed to be determined by the resolved shear stress $\tau_{(k)}^{(r)}=\boldsymbol{\sigma} \cdot \boldsymbol{\mu}_{(k)}^{(r)}$ via:

$\gamma_{(k)}^{(r)}=\frac{\partial \phi_{(k)}^{(r)}}{\partial \tau}\left(\tau_{(k)}^{(r)}\right)$,

where the convex function $\phi_{(k)}^{(r)}$ characterizes the response of the slip system, and $\boldsymbol{\mu}_{(k)}^{(r)}=1 / 2\left(\mathbf{m}_{(k)}^{(r)} \otimes\right.$ $\left.\mathbf{n}_{(k)}^{(r)}+\mathbf{n}_{(k)}^{(r)} \otimes \mathbf{m}_{(k)}^{(r)}\right)$ is the corresponding Schmid tensor, with $\mathbf{n}_{(k)}^{(r)}$ and $\mathbf{m}_{(k)}^{(r)}$ denoting the unit vectors normal to the slip plane and along the slip direction of the system, respectively. Note that $\boldsymbol{\mu}_{(k)}^{(r)}$ is related to the corresponding tensor $\boldsymbol{\mu}_{(k)}$ for a reference crystal via $\boldsymbol{\mu}_{(k)}^{(r)}=\mathbf{Q}^{(r) T}$ $\boldsymbol{\mu}_{(k)} \mathbf{Q}^{(r)}$. The slip potential $\phi_{(k)}^{(r)}$ depends on the slip mechanism and on slip history, which may vary with crystal orientation if strain hardening is orientationdependent. The Eulerian strain rate $\varepsilon^{(r)}$ in the crystal is obtained by summing up all slips, and the local constitutive response in the polycrystal is then defined:

$$
\varepsilon^{(r)}=\sum_{k=1}^{K} \gamma_{(k)}^{(r)} \boldsymbol{\mu}_{(k)}^{(r)} \text { and } \varepsilon(x)=\sum_{r=1}^{N} \chi^{(r)}(x) \varepsilon^{(r)}=\frac{\partial u}{\partial \boldsymbol{\sigma}},
$$

where $\varepsilon$ denotes the field of Eulerian strain rate. The stress potential $u$ equals $u^{(r)}$ when $\mathbf{x}$ is in a single crystal with orientation $\mathbf{Q}^{(r)}$ :

$$
u(\mathbf{x}, \boldsymbol{\sigma})=\sum_{r=1}^{N} \chi^{(r)}(\mathbf{x}) u^{(r)}(\boldsymbol{\sigma})
$$

with

$$
u^{(r)}(\boldsymbol{\sigma})=\sum_{k=1}^{K} \phi_{(k)}^{(r)}\left(\tau_{(k)}^{(r)}\right) .
$$

It is known (e.g., Hutchinson, 1976; Ponte Castañeda and Suquet, 1998) that the effective viscous response for the polycrystal may be written in the form:

$$
\begin{aligned}
\bar{\varepsilon}=\frac{\partial \tilde{U}}{\partial \overline{\boldsymbol{\sigma}}}, \quad \tilde{U}(\overline{\boldsymbol{\sigma}}) & =\min _{\boldsymbol{\sigma} \epsilon S(\overline{\boldsymbol{\sigma}})}\langle u(\mathbf{x}, \boldsymbol{\sigma})\rangle \\
& =\min _{\boldsymbol{\sigma} \epsilon S(\bar{\sigma})} \sum_{r=1}^{N} c^{(r)}\langle u(\mathbf{x}, \boldsymbol{\sigma})\rangle^{(r)},
\end{aligned}
$$

where $\tilde{U}$ is the effective stress potential for the polycrystal. In this relation, $\bar{\varepsilon}=\langle\varepsilon\rangle$ is the average strain rate, and $S(\overline{\boldsymbol{\sigma}})$ is the set of statically admissible stress fields such that $\operatorname{div} \boldsymbol{\sigma}=\mathbf{0}$ in $\Omega$ and $h\langle\boldsymbol{\sigma}\rangle=\overline{\boldsymbol{\sigma}}$. In general, the exact effective stress potential $\tilde{U}(\overline{\boldsymbol{\sigma}})$ for nonlinear heterogeneous materials cannot be obtained unless both the response of the slip systems and the microstructure are very simple. An alternative approach is to evaluate bounds for $\tilde{U}$, for instance the Reuss uniform-stress lower bound, the Taylor uniform-strainrate upper bound and the more recent variational selfconsistent upper bound. Estimates for $\tilde{U}(\overline{\boldsymbol{\sigma}})$ can also be defined, such as the incremental, tangent, variational and, more recently, second-order self-consistent estimates. Since the latter has been described thoroughly by Liu and Ponte Castañeda (2004a), only the essential formulae are recalled, adding some relations that are required to compute texture evolution.

The second-order estimate of the effective stress potential for a polycrystal loaded with a macroscopic stress $\overline{\boldsymbol{\sigma}}$ is given by:

$$
\begin{aligned}
\tilde{U}(\overline{\boldsymbol{\sigma}})= & \sum_{r=1}^{N} \sum_{k=1}^{K} c^{(r)}\left\{\phi_{(k)}^{(r)}\left(\hat{\tau}_{(k)}^{(r)}\right)\right. \\
& \left.+\phi_{(k)}^{(r)^{\prime}}\left(\bar{\tau}_{(k)}^{(r)}\right)\left(\bar{\tau}_{(k)}^{(r)}-\hat{\tau}_{(k)}^{(r)}\right)\right\},
\end{aligned}
$$

where $\phi_{(k)}^{(r)^{\prime}}$ denotes the derivative of $\phi_{(k)}^{(r)}, \bar{\tau}_{(k)}^{(r)}$ and $\hat{\tau}_{(k)}^{(r)}$ are computed from the averages and second moments of stress in the linear comparison composite (see Liu, 2003, for details), and are required to satisfy the "generalized secant" condition:

$\phi_{(k)}^{(r)^{\prime}}\left(\hat{\tau}_{(k)}^{(r)}\right)-\phi_{(k)}^{(r)^{\prime}}\left(\bar{\tau}_{(k)}^{(r)}\right)=\alpha_{(k)}^{(r)}\left(\hat{\tau}_{(k)}^{(r)}-\bar{\tau}_{(k)}^{(r)}\right)$,

where the $\alpha_{(k)}^{(r)}$ variables define the compliances in the grains of the linear comparison polycrystal. The latter has the same microstructure as the associated non- 
linear polycrystal, but differs from the latter by a local linear thermoviscous behavior.

As discussed by Liu and Ponte Castañeda (2004a), the "energy" estimate (5) has the advantage of being exact to the second-order in the heterogeneity contrast, but it does not provide an explicit constitutive relation connecting the average stress $\bar{\sigma}$ and strain rate $\bar{\varepsilon}$. In other words, the effective stress-strain-rate relation for the polycrystal must be computed by differentiating numerically the relation (5), according to Eq. (4). A simpler "constitutive" (or generalized "affine") alternative, which is unfortunately not exact to second-order in the contrast, but still reasonably close to the just described estimate, is to make use of the effective stress-strain-rate relations for the linear comparison polycrystal. Such an estimate can be shown to reduce to the following expression:

$\bar{\varepsilon}=\sum_{r=1}^{N} \sum_{k=1}^{K} c^{(r)} \bar{\gamma}_{(k)}^{(r)} \boldsymbol{\mu}_{(k)}^{(r)}$

where

$\bar{\gamma}_{(k)}^{(r)}=\phi_{(k)}^{(r)^{\prime}}\left(\bar{\tau}_{(k)}^{(r)}\right)$

are the average slip rates in grains with orientation $r$.

Correspondingly, the average strain rate in the crystals of orientation $r$ can be shown to be given by expressions:

$\bar{\varepsilon}^{(r)}=\sum_{k=1}^{K} \bar{\gamma}_{(k)}^{(r)} \boldsymbol{\mu}_{(k)}^{(r)}$

Finally, the average continuum spin $\overline{\boldsymbol{\omega}}^{(r)}$ in grains with orientation $r$ is related to the mean spin $\bar{\omega}$ and the strain rate $\bar{\varepsilon}$ of the polycrystal through:

$\overline{\boldsymbol{\omega}}^{(r)}=\overline{\boldsymbol{\omega}}-\mathbf{R} \mathbf{P}^{-1}\left(\bar{\varepsilon}-\bar{\varepsilon}^{(r)}\right)$,

where $\mathbf{R}$ and $\mathbf{P}$ are microstructural tensors (see Liu, 2003, for details). It is emphasized that tensors in (10), i.e. $\mathbf{R}, \mathbf{P}$ and the strain rate $\bar{\varepsilon}^{(r)}$, refer to the linear comparison composite and depend on the homogenization model utilized. For the Taylor model, for instance, where assumption $\bar{\varepsilon}^{(r)}=\bar{\varepsilon}$ is taken, the average continuum spin is uniform throughout the polycrystal and equal to $\bar{\omega}$.

\subsection{Microstructure evolution}

The evolution of the granular microstructure of polycrystals will be assumed to be controlled by the mean flow (Ponte Castañeda, 1997). In other words, the shape and orientation of the grains are assumed to be controlled by the average strain rate $\bar{\varepsilon}$ and spin $\bar{\omega}$ in the polycrystal, which is the simplest hypothesis that is consistent with preservation of the polycrystal integrity. More complete descriptions incorporating local grain effects are certainly possible, but at the expense of having to introduce and keep track of a much larger set of two-point statistical variables. In addition, it is also necessary to keep track of the orientation of the atomic lattice within each grain, which will be assumed here to be controlled by the average spin $\bar{\omega}^{(r)}$ of the material within the grains. It is emphasized that the orientation of the grains (the morphological texture) and that of the atomic lattice of the material inside the grains (the crystallographic texture) are different in general (Lipinski et al., 1990).

For the evolution of the morphological texture, it is reasonable to assume that the average shape of all grains deforms from and to ellipsoidal shapes, as controlled by the average strain rate $\bar{\varepsilon}$ and spin $\bar{\omega}$. Thus, the aspect ratios $w_{1}^{(\mathrm{g})}$ and $w_{2}^{(\mathrm{g})}$ (keeping $w_{3}^{(\mathrm{g})}=1$ as a reference) are governed by the kinematical relations:

$\dot{w}_{1}^{(\mathrm{g})}=w_{1}^{(\mathrm{g})}\left(\bar{\varepsilon}_{33}^{\prime}-\bar{\varepsilon}_{11}^{\prime}\right)$

and

$\dot{w}_{2}^{(\mathrm{g})}=w_{2}^{(\mathrm{g})}\left(\bar{\varepsilon}_{33}^{\prime}-\bar{\varepsilon}_{22}^{\prime}\right)$,

where the primes in this subsection denote tensor components relative to coordinates that are instantaneously aligned with the principal axes of the ellipsoidal grains. The principal axes of the morphological texture are related to the sample (polycrystal) coordinates by an orientation tensor $\mathbf{G}^{(\mathrm{g})}$, which is a function of three Euler angles $\left(\varphi_{1}^{(\mathrm{g})}, \phi^{(\mathrm{g})}, \varphi_{2}^{(\mathrm{g})}\right)$, where Bunge's convention for Euler angles is utilized. The expression for $\mathbf{G}^{(\mathrm{g})}$ is standard and has the following matrix form (see Bunge and Esling, 1982; Nebozhyn, 2000):

$\mathbf{G}^{(\mathrm{g})}=\left[\begin{array}{lll}C_{1} C_{2}-S_{1} S_{2} C & S_{1} C_{2}+C_{1} S_{2} C & S_{2} S \\ -C_{1} S_{2}-S_{1} C_{2} C & -S_{1} S_{2}+C_{1} C_{2} C & C_{2} S \\ S_{1} S & -C_{1} S & C\end{array}\right]$, 
with $C_{1}=\cos \varphi_{1}^{(\mathrm{g})}, C=\cos \phi^{(\mathrm{g})}, C_{2}=\cos \varphi_{2}^{(\mathrm{g})}, S_{1}=\sin$ $\varphi_{1}^{(\mathrm{g})}, S=\sin \phi^{(\mathrm{g})}, S_{2}=\sin \varphi_{2}^{(\mathrm{g})}$. The orientation change of the morphological texture is thus described by the evolution of the orientation tensor $\mathbf{G}^{(\mathrm{g})}$ :

$\dot{\mathbf{G}}^{(\mathrm{g})}=-\mathbf{G}^{(\mathrm{g})} \overline{\boldsymbol{\omega}}^{(\mathrm{g})}$,

where the spin tensor $\bar{\omega}^{(\mathrm{g})}$ is due to the average strain rate $\bar{\varepsilon}$ and spin $\bar{\omega}$ in the polycrystal, and its nonzero components are calculated from the following relations:

$$
\begin{aligned}
\left(1-\frac{\left(w_{l}^{(\mathrm{g})}\right)^{2}}{\left(w_{k}^{(\mathrm{g})}\right)^{2}}\right) \bar{\omega}_{k l}^{\mathrm{g} \prime}= & \left(1-\frac{\left(w_{l}^{(\mathrm{g})}\right)^{2}}{\left(w_{k}^{(\mathrm{g})}\right)^{2}}\right)^{\prime}{ }_{k l}^{\prime} \\
& +\left(1+\frac{\left(w_{l}^{(\mathrm{g})}\right)^{2}}{\left(w_{k}^{(\mathrm{g})}\right)^{2}} \overline{\bar{\varepsilon}}_{k l}^{\prime},\right.
\end{aligned}
$$

with $k \neq l$, and $w_{k}^{(\mathrm{g})}$ denoting the current value of the aspect ratios. Eq. (14) is identical to well-known kinematical relations given by Ogden (1984):

$\bar{\omega}_{k l}^{(\mathrm{g}) \prime}=\bar{\omega}_{k l}^{\prime}+\frac{\left(w_{k}^{(\mathrm{g})}\right)^{2}+\left(w_{l}^{(\mathrm{g})}\right)^{2}}{\left(w_{k}^{(\mathrm{g})}\right)^{2}-\left(w_{l}^{(\mathrm{g})}\right)^{2}} \bar{\varepsilon}_{k l}^{\prime}$

when none of the $w^{(\mathrm{g})}$ is equal. In the case when a pair of $w^{(\mathrm{g})}$ are equal, for example when $w_{k}^{(\mathrm{g})}=w_{l}^{(\mathrm{g})}(k \neq l)$, relation (14) has a clearer interpretation, i.e. the principal axes should be well selected so that $\bar{\varepsilon}_{k l}^{\prime}=0$, and $\bar{\omega}_{k l}^{(\mathrm{g}) \prime}=\bar{\omega}_{k l}^{\prime}$. This treatment is consistent with the remarks by Kocks et al. (1998) on the "Eulerian spin". Note that when the grains are equiaxed, $w_{1}^{(\mathrm{g})}=w_{2}^{(\mathrm{g})}=w_{3}^{(\mathrm{g})}$, it follows that $\overline{\boldsymbol{\omega}}^{(\mathrm{g})}=\overline{\boldsymbol{\omega}}$.

Similarly, the crystallographic orientation of the lattice inside the $r$-th grain is described by an orientation tensor $\mathbf{Q}^{(r)}$, whose expression is the same as for the grain orientation $\mathbf{G}^{(\mathrm{g})}$ but with the morphological angles $\left(\varphi_{1}^{(\mathrm{g})}, \phi^{(\mathrm{g})}, \varphi_{2}^{(\mathrm{g})}\right)$ replaced by the crystallographic angles $\left(\varphi_{1}^{(r)}, \phi^{(r)}, \varphi_{2}^{(r)}\right)$. The evolution of $\mathbf{Q}^{(r)}$ is governed by the "microstructural" spin, which is given by the difference between the average continuum spin $\bar{\omega}^{(r)}$ and the average plastic spin $\bar{\omega}_{\mathrm{pl}}^{(r)}$ in the grain (see Mandel, 1972):

$\dot{\mathbf{Q}}^{(r)}=\mathbf{Q}^{(r)}\left(\overline{\boldsymbol{\omega}}^{(r)}-\overline{\boldsymbol{\omega}}_{\mathrm{pl}}^{(r)}\right)$, where $\bar{\omega}^{(r)}$ is given by (10), and $\bar{\omega}_{\mathrm{pl}}^{(r)}$ results from the intragrain lattice slips:

$\overline{\boldsymbol{\omega}}_{\mathrm{pl}}^{(r)}=\frac{1}{2} \sum_{k=1}^{K} \gamma_{(k)}^{(r)}\left(\mathbf{m}_{(k)}^{(r)} \otimes \mathbf{n}_{(k)}^{(r)}-\mathbf{n}_{(k)}^{(r)} \otimes \mathbf{m}_{(k)}^{(r)}\right)$.

Here the $\bar{\gamma}_{(k)}^{(r)}$ are the average slip rates estimated consistently from (8) for the second-order self-consistent model.

It is emphasized that the orthogonality of the orientation tensors $\mathbf{G}^{(\mathrm{g})}$ and $\mathbf{Q}^{(\mathrm{r})}$ must be preserved. This requires a properly selected numerical scheme for the time integration of differential Eqs. (13) and (16). Here, we make use of the following relation for a skew-symmetric tensor $\mathbf{A}$ (i.e. $\mathbf{A}^{\mathrm{T}}=-\mathbf{A}$ ),

$\exp \mathbf{A}=\mathbf{I}+\frac{\sin a}{a} \mathbf{A}+\frac{1-\cos a}{a^{2}} \mathbf{A}^{2}$

with

$a=\sqrt{\frac{\mathbf{A}: \mathbf{A}}{2}}$,

which is known as Rodrigues' formula, such that the grain orientation $\mathbf{G}^{(\mathrm{g})}$ at time $t+\delta t$ is calculated with

$\mathbf{G}^{(g)}(t+\delta t)=\mathbf{G}^{(\mathrm{g})}(t) \exp \left(-\overline{\boldsymbol{\omega}}^{(\mathrm{g})} \delta t\right)$,

and a similar equation applies for the evolution of the crystallographic orientation tensors $\mathbf{Q}^{(r)}$.

\section{Single-crystal constitutive behavior and initial texture}

\subsection{Slip behavior and hardening law for halite}

Halite is an aggregate of ionic crystals with a cubic structure and is assumed to deform, at room temperature, by crystallographic slip on three families of systems: six A $(\{110\}\langle 110\rangle)$ systems, six B $(\{100\}\langle 110\rangle)$ systems and twelve $C(\{111\}\langle 110\rangle$, the same as in facecentered cubic crystals) systems. Their constitutive behavior is governed by a power-law slip potential:

$\phi_{(k)}^{(r)}(\tau)=\frac{1}{1+n} \tau_{0(k)}^{(r)} \gamma_{0}\left(\frac{|\tau|}{\tau_{0(k)}^{(r)}}\right)^{1+n}$. 
The parameter $n(=1 / m)$ defines the nonlinearity and depends on temperature (Carter and Heard, 1970). At room temperature $\left(20^{\circ} \mathrm{C}\right)$, an exponent of $n=10$ has been suggested and used in the HEP simulations of Lebensohn et al. (2003). This value is close to the value of 10.5 suggested by Carter and Heard (1970) for the A systems, which are the most active. Here, the value of 10 will be used in the calculations to follow in order to be able to compare with the HEP simulations. The reference slip rate $\gamma_{0}$ is set equal to $10^{-5} \mathrm{~s}^{-1}$. The reference resolved shear stresses (RRSS), $\tau_{0(k)}^{(r)}$, are different in general for the three families of slip systems. In this work, the A systems are assumed to be "soft" and the B and C systems are "hard". At the undeformed state, the RRSS are assumed to be 4.8 MPa for A systems and 19.2 MPa, i.e. four times harder, for B and C systems. These values are very different from the material parameters employed in our previous simulations for halite (Liu, 2003), and correspond to a more anisotropic slip behavior, since the A family has only 2 linearly independent slip systems.

Furthermore, a phenomenological Voce-type hardening law (see Balasubramanian and Anand, 2002) was adopted in Lebensohn et al. (2003) and will be employed in this work without modification:

$\dot{\tau}_{0(k)}^{(r)}(t)=H \frac{\tau_{(k)}^{\mathrm{sat}}-\tau_{0(k)}^{(r)}(t)}{\tau_{(k)}^{\mathrm{sat}}-\tau_{0(k)}^{(r)}(0)} \sum_{l=1}^{24}\left|\frac{\gamma_{(l)}^{(r)}}{\gamma_{0}}\right|$,

where $H=105 \mathrm{MPa}$ is the initial hardening parameter, $\tau_{(k)}^{\text {sat }}$ are saturation stresses and are set equal to 9.1, 36.4 and $36.4 \mathrm{MPa}$ for the families $\mathrm{A}, \mathrm{B}$ and $\mathrm{C}$, respectively. Note that the summation above is taken over all slip systems in a grain. It should further be noted that all material parameters above, that are listed in Table 1, were selected for the best agreement between the HEP prediction of the stress-strain

Table 1

Halite single crystal parameters at $20{ }^{\circ} \mathrm{C}$

\begin{tabular}{llll}
\hline & $\mathrm{A}(\{110\}\langle 110\rangle)$ & $\mathrm{B}(\{100\}\langle 110\rangle)$ & $\mathrm{C}(\{111\}\langle 110\rangle)$ \\
\hline$m=1 / n$ & 0.1 & 0.1 & 0.1 \\
$\gamma_{0}$ & 0.00001 & 0.00001 & 0.00001 \\
$H$ & 105 & 105 & 105 \\
$\tau_{0}$ & 4.8 & 19.2 & 19.2 \\
$\tau^{\text {sat }}$ & 9.1 & 36.4 & 36.4 \\
\hline
\end{tabular}

Data taken from Lebensohn et al. (2003). $\tau$ 's and $H$ are in MPa, $\gamma_{0}$ is in $\mathrm{s}^{-1}$. response and the experimental results (Lebensohn et al., 2003), and they are possibly not optimized for other simulations. Nevertheless, use will be made of these parameters, as the main purpose in this work is to investigate the model predictions for texture evolution, which should not be too sensitive to the hardening parameters for high-contrast polycrystals (Liu et al., 2003).

\subsection{Experiments and initial texture}

Recent experiments were performed by loading polycrystalline halite samples in a triaxial multianvil pressure apparatus (see Kern, 1979, for details). The applied stress was of the following type (Lebensohn et al., 2003):

$\overline{\boldsymbol{\sigma}}=\sigma_{1}\left(\mathbf{e}_{1} \otimes \mathbf{e}_{1}+\mathbf{e}_{2} \otimes \mathbf{e}_{2}\right)+\sigma_{3} \mathbf{e}_{3} \otimes \mathbf{e}_{3}$,

where $\mathbf{e}_{3}$ refers to the extension direction, and both $\sigma_{1}$ and $\sigma_{3}$ have negative values. The samples were preloaded with $\sigma_{1}=\sigma_{3}=-50 \mathrm{MPa}$, and then deformed by increasing the magnitude of the lateral stress $\sigma_{1}$ and adjusting the latter value such that a constant axial strain rate of $10^{-5} \mathrm{~s}^{-1}$ was obtained. The polycrystalline halite specimens were prepared from rocksalt powder by compaction, without annealing. In this work, the polycrystalline samples are assumed to be fully isotropic initially, i.e. the grains are statistically equiaxed $\left(w_{1}^{(\mathrm{g})}(0)=w_{2}^{(\mathrm{g})}(0)=1\right)$ and the ODF for the crystallographic texture is uniform. Because of the available computer power and the numerical efficiency of the yet-to-be-optimized program for the second-order method, a minimum set of discrete crystal orientations is desired. Since the material is initially isotropic and deforms in an axially symmetric manner, use has been made of 45 equally weighted and uniformly distributed discrete orientations in a stereographic triangle, as shown in Fig. 1a, where equal-area projections are used, as in all pole figures shown in this paper. Contributions from the orientations outside the triangle are obtained by proper symmetrization of the averages over the triangle (Nebozhyn, 2000), and it has been checked that a sufficient accuracy was obtained for the effective flow stress and field quantities in undeformed FCC cubic crystals (Lebensohn et al., 2004b). It should be pointed out, however, that this is a much smaller set of representative orientations than in the HEP numerical 
(a)

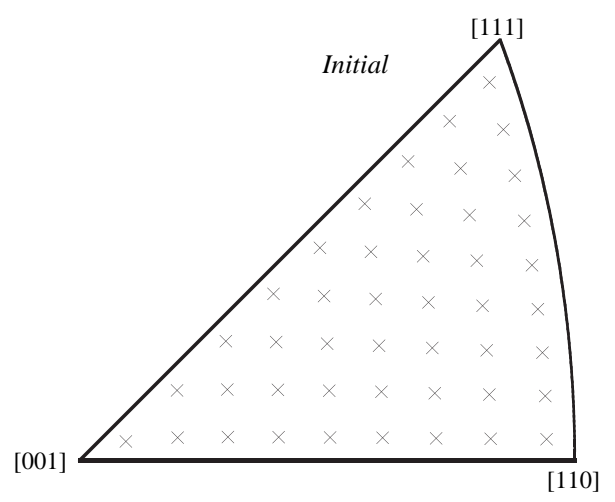

(b)

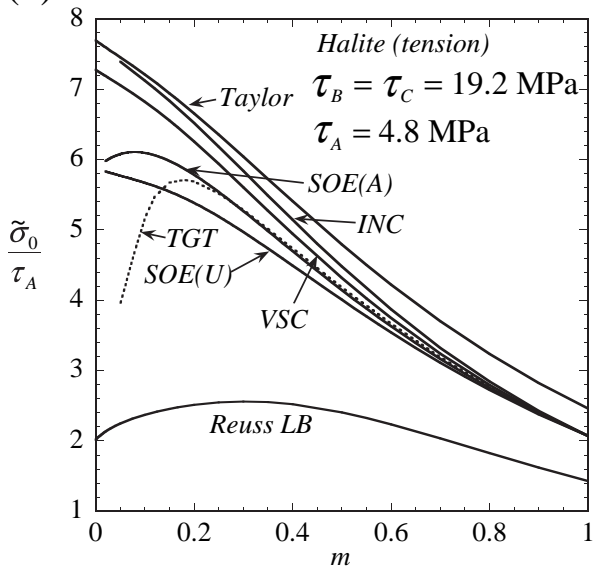

Fig. 1. (a) Inverse pole figure of the initial texture of the isotropic halite polycrystal, using 45 equally weighted and uniformly distributed orientations in a stereographic triangle. (b) Effective flow stress $\tilde{\sigma}_{0}$ for untextured, power-law halite-type polycrystals with equiaxed grains loaded in uniaxial tension, as function of the strain-rate sensitivity $m$ and normalized by the reference resolved shear stress for systems A.

simulation of Lebensohn et al. (2003), where 4096 randomly distributed orientations were used, and thus is not appropriate for a very accurate description of deformation texture. Nevertheless, the choice is a compromise between accuracy and efficiency, which will allow comparisons between different estimates.

It should be emphasized at this stage that halite polycrystals can behave very differently depending on water content and confining pressure, i.e. with or without fluidassisted grain boundary migration (cf. Watanabe and Peach, 2002; Ter Heege et al., in press). Obviously, these effects can have a major influence on the microstructural evolution including texture patterns, as well as on the macroscopic response of the polycrystal. In this work, again consistent with the work of Lebensohn et al. (2003), the assumption is made that the halite polycrystal is under "dry" conditions, so that dynamic recrystallization can be neglected.

\section{Initial response of the polycrystal and texture evolution}

\subsection{The effective flow stress of untextured polycrystals}

Comparisons are first carried out between the Taylor, tangent, variational, and second-order self-consis- tent estimates for the effective flow stress in untextured halite-type polycrystals under axisymmetric loading, taking the same reference resolved shear stresses as in Table 1, but different rate-sensitivity exponents $m$ ranging from 1 (linear slip) to 0 (rate-insensitive limit). For isotropic power-law polycrystals, the effective flow stress $\tilde{\sigma}_{0}$ is defined from the effective stress potential $\tilde{U}(\overline{\boldsymbol{\sigma}})(4)$ via

$\tilde{U}(\overline{\boldsymbol{\sigma}})=\frac{1}{1+n} \tilde{\sigma}_{0} \gamma_{0}\left(\frac{\overline{\boldsymbol{\sigma}}_{e}}{\tilde{\sigma}_{0}}\right)^{1+n}$,

where $\bar{\sigma}_{e}=\sqrt{\frac{3}{2} \overline{\mathbf{s}} \cdot \overline{\mathbf{s}}}$ is the effective von Mises equivalent stress, and $\overline{\mathbf{s}}=\overline{\boldsymbol{\sigma}}-1 / 3(\operatorname{tr} \overline{\boldsymbol{\sigma}}) \mathbf{I}$. The second-order predictions for the effective flow stress are given with two versions, namely the "energy" estimate, associated with (5) and denoted $\mathrm{SOE}(\mathrm{U})$ in Fig. 1b, and the "constitutive" estimate, which is calculated from the stress-strain rate relation (7) with an effective strain rate $\bar{\varepsilon}=-1 / 2 \gamma_{0}\left(\mathbf{e}_{1} \otimes \mathbf{e}_{1}+\mathbf{e}_{2} \otimes \mathbf{e}_{2}\right)+\gamma_{0} \mathbf{e}_{3} \otimes \mathbf{e}_{3}$, and denoted SOE(A). In this work, the short-hand notations VSC, SOE and TGT denote variational, second-order and tangent self-consistent results, respectively. For reference, the incremental self-consistent (INC) (Hill, 1965; Hutchinson, 1976) and Reuss (Reuss, 1929) estimates are also shown in Fig. 1b. The effective flow stresses are plotted as functions of $m$, and are normalized by the initial reference resolved shear stress of the A systems, 
$\tau_{\mathrm{A}}$. The various self-consistent estimates start from the same value for the linear case $(m=1)$, and diverge as the slip nonlinearity increases. The incremental estimate, which is much lower than the Taylor upper bound at $m=1$, tends to the latter in the rate-insensitive limit $(m \rightarrow 0)$. The variational self-consistent estimate is lower than the Taylor upper bound by about $0.4 \tau_{\mathrm{A}}$ for all rate-sensitivity exponents $m$. The second-order estimates are softer than the VSC result, as they should, since the latter sets a rigorous upper bound for all selfconsistent estimates. There is a "duality gap" (Liu and Ponte Castañeda, 2004a) between the second-order estimates, with the "constitutive" estimate being somewhat higher than the corresponding "energy" version for $m<1$. However, the gap seems to vanish in the rateinsensitive limit, as was found for other polycrystals (Liu and Ponte Castañeda, 2004a,b). At the rate-insensitive limit, the second-order estimates tend to about $5.85 \tau_{\mathrm{A}}$, which is $1.4 \tau_{\mathrm{A}}$ softer than the VSC result and is considerably stiffer than the Reuss lower bound. On the other hand, the tangent self-consistent estimate agrees with the SOE results for weakly nonlinear cases when $m \geq 0.3$, and falls quickly to the Reuss lower bound as $m \rightarrow 0$. At the rate sensitivity that applies for halite, i.e. $m=0.1$, the TGT result is dropping and is below the "constitutive" version of the SOE estimate by about $0.9 \tau_{\mathrm{A}}$ or $4.3 \mathrm{MPa}$. In comparison with the HEP result (Lebensohn et al., 2003) at zero axial strain, which is about $28 \mathrm{MPa}$, the $\operatorname{SOE}(\mathrm{A})(\sim 29.2$ $\mathrm{MPa})$ stands out as the most accurate one. It should be noted that the HEP computation referred to here employed a one-element-per-grain discretization (Beaudoin et al., 1995), and thus necessarily leads to an overly stiff stress-strain response (because the discretization is not sufficient in the context of the relevant variational principle). In other words, the energy version of the SOE, denoted SOE(U) in the plots, could be more accurate. However, as already mentioned, the stress-strain relation associated with the second-order "energy" estimate (5) has to be computed by means of numerical differentiations, and, for this reason, the "constitutive" version will be employed in the simulation of texture evolution below, and denoted as "plain" SOE.

\subsection{Texture evolution}

The simulation of texture evolution in polycrystals is achieved incrementally. At each increment, the instantaneous response in the polycrystal induced by an increment of external loading is evaluated first, with fixed material parameters and microstructural variables. In this step, the macroscopic stress-strain relation and the average slip rates are estimated by applying the homogenization models. The increment ends with the updating of the reference resolved shear stresses, $\tau_{\mathrm{A}}, \tau_{\mathrm{B}}$ and $\tau_{\mathrm{C}}$, and the microstructural variables, $w_{1}^{(\mathrm{g})}, w_{2}^{(\mathrm{g})}$ and $\mathbf{Q}^{(\mathrm{r})}$ by using the hardening law (21) and the kinematics relations (11) and (16), respectively. For symmetry reasons, in the simulation of the experimental tests considered, we applied either an axisymmetric stress, $\overline{\boldsymbol{\sigma}}=-1 / 2 \sigma\left(\mathbf{e}_{1} \otimes \mathbf{e}_{1}+\mathbf{e}_{2} \otimes\right.$ $\left.\mathbf{e}_{2}\right)+\sigma \mathbf{e}_{3} \otimes \mathbf{e}_{3}$, which is actually equivalent to the loading (22) applied in the experiments, since a hydrostatic pressure does not affect viscoplastic polycrystals, or an axisymmetric strain. The use of a strain condition is more convenient for most homogenization models, but the version of the second-order method that we use requires applying a stress, as mentioned in the previous section. Consequently, $\sigma$ increments were adjusted during the SOE simulations in order to achieve a constant axial strain rate of $10^{-5} \mathrm{~s}^{-1}$.

All homogenization simulations, except for those arising from the second-order method, used similar increments in the axial strain, which correspond to 60 equal steps for the extension test up to $30 \%$ axial strain. Finer incremental steps have been tested, but did not yield significant effects on the stress-strain relation and the texture. The secondorder method, which involves solving a large set of highly nonlinear equations, needs much finer strain increments in order to ensure convergence. Axial strain steps of $10^{-4}$ are required in the early stage of deformation, when the material properties evolve rapidly. After that, the RRSS are closer to their saturation values, and larger steps are possible, yet still finer than those for the other models. As a result, the time required for a second-order simulation using 45 representative orientations is about three days on a computer equipped with a $2.8 \mathrm{GHz}$ Pentium IV processor. Simulations were also attempted with a set of 135 orientations showing that much finer steps were needed, with each step requiring at least 9 times longer than with 45 orientations. 


\subsection{Macroscopic stress-strain relations and relative activities in tension}

Fig. 2a presents the macroscopic stress-strain responses predicted by the Taylor, variational (VSC), second-order (SOE) and tangent (TGT) self-consistent methods, together with the corresponding "hybrid element polycrystal" (HEP) results and experimental measurements of Lebensohn et al. (2003). As expected, the Taylor model predicts the highest stress-strain curve, followed by the VSC, HEP and SOE models in order, and with the TGT estimate being the lowest. The TGT estimate increases rapidly for small strain, like the others, and reaches a plateau for axial strains larger than $15 \%$. The other models all capture the general shape of the stress-strain curve given by the HEP simulation, with different magnitudes. The general shape of these curves can be compared with what was obtained by Wenk et al. (1989) for the Taylor and TGT models: without strain hardening, the effective stress was found almost constant, while a linear strain hardening induced an almost linearly increasing effective stress. This suggests that the stress-strain response of halite in axisymmetric extension reflects directly the strain hardening law and is uncoupled from the texture evolution, which is a remarkable feature. In the present case of a saturating hardening, a decreasing slope of the stress-strain curve is observed, with the
SOE model giving the largest slope after $30 \%$ extension. It is thus speculated that all these homogenization models, with the possible exception of the tangent model, could predict stress-strain curves that agree with the HEP and experimental results, by suitably choosing the crystal parameters. On the other hand, the upper-bound nature of the variational self-consistent estimate implies that its closest but lower neighbor, the HEP result, could also be too stiff for the polycrystal. Consequently, the material parameters used in the simulations (Table 1) could be underestimates for the actual values. In other words, the secondorder estimates would be expected to possibly provide an even better agreement with the experimental results if more accurate value for the material parameters were available.

The various models that are applied here predict different slip rates on the slip systems that are available in the polycrystal, and one method for comparing the results consists in computing the relative activities of the families of slip systems. The relative activity is defined as the sum of the slip rates obtained on all the systems that belong to a given family, whatever the grain orientation they belong to, divided by the sum over all the available systems. Fig. $2 b$ shows the development of the relative activity of A systems during deformation (the HEP data was provided by R.A. Lebensohn). For all models, the $\mathrm{C}$ systems yield (a)

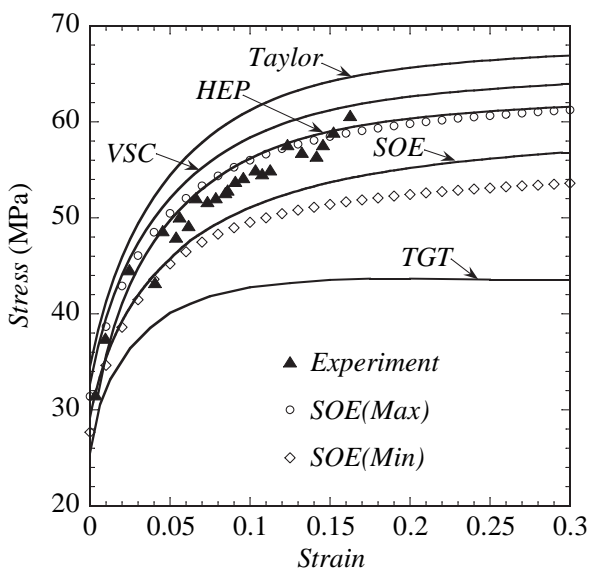

(b)

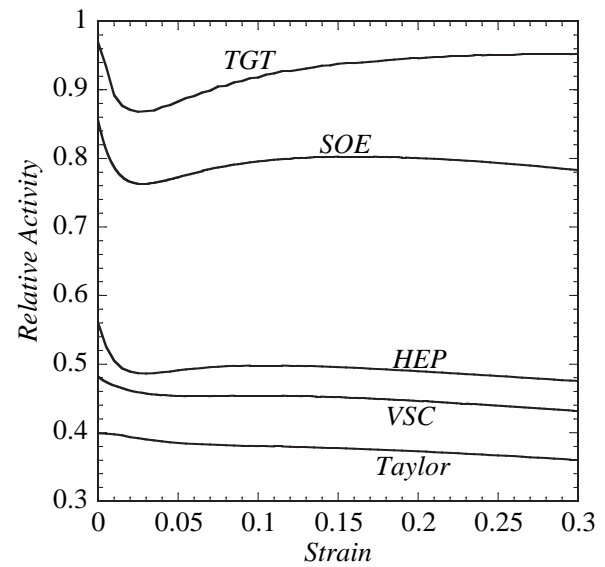

Fig. 2. Macroscopic stress-strain relation and relative activity for uniaxial extension of halite at room temperature. Comparison of the HEP simulations and experimental results of Lebensohn et al. (2003) with the Taylor, variational (VSC), second-order (SOE) and tangent (TGT) selfconsistent estimates. (a) Macroscopic stress-strain relation, where SOE (Max) and SOE (Min) denote the highest and lowest SOE values obtained when using various initial sets of 45 random orientations. (b) Evolution of the relative activity of A systems. 
such little slip that the relative activity of the B systems is just slightly lower than one minus that of the A systems. It may first be observed that the order of the curves in Fig. 2a reflects exactly the order of the activity of the B (hard) systems, even when strain hardening develops and texture evolves. It may also be noted that SOE shows a distinctive feature: the activity of the A systems is much closer to the TGT value than to the HEP results, although the opposite applies to the stress-strain curves. For the TGT and SOE models, as well as for the HEP simulations, the total activity on A systems drops during the early stage of deformation, then flips up smoothly after about $2.5 \%$ axial strain, and starts decreasing slowly after $15 \%(10 \%)$ strain for SOE (HEP), but not for TGT. At the other extreme, the Taylor model gives a steadily and slowly decreasing activity. The initial reduction may be explained by the hardening behavior at the grain level and the sensitivity of the model to the contrast, namely the ratio between the reference resolved shear stresses (RRSS) for the hard (B and C) and soft (A) systems. At the early stage of deformation, the hardening law (21) increases the RRSS quickly for all slip systems in a grain by approximately the same amount, and hence reduces the contrast. For instance, the contrast for most grains, that starts from a value of 4 (Table 1), is found in the VSC and SOE simulations to reach the lowest value, about 3.0 , at approximately $2 \%$ axial strain, and turns back to 3.8 and more after $15 \%$ strain (recall the contrast for saturation values is 4 , Table 1). Thus the tangent model, that favors the soft A systems, starts activating some additional B systems. Moreover, the hardening is rapidly saturated by the intense activity associated with the small number of active systems in the TGT model, which may explain the plateau shown in Fig. 2a. The Taylor model, on the other hand, requires most A and B systems to be activated simultaneously in order to accommodate an arbitrary strain rate, and consequently predicts very stable relative activity for all systems and a slower saturation of strain hardening, as has been found in previous simulations on titanium (Liu et al., 2003).

\subsection{Texture evolution in axial extension}

In order to interpret properly the texture-evolution predictions in halite, it is important to recall that the rotation of the crystallographic axes is controlled by Eq. (16), where the average continuum spin is given by Eq. (10) and the plastic spin by Eq. (17). In the present simulation, the overall spin $\bar{\omega}$ is 0 , and thus the average continuum spin $\bar{\omega}(r)$ is determined by the second term of Eq. (10) only (except for the Taylor model, where it is zero), where $\mathbf{R}$ and $\mathbf{P}$ vary when both the morphological and the crystallographic textures evolve. It should be noted that the version of the tangent model that we use in this paper assumes all grains have the same shape, for consistency with the theory presented above, whereas different grain shapes develop for different crystallographic orientations in the simulations of Wenk et al. (1989) and Lebensohn et al. (2003). This should not lead to significant differences in the moderate range of strain considered in this paper. It may also be recalled that the A systems do not contribute to the plastic spin: as stressed by Wenk et al. (1989) and Lebensohn et al. (2003), each system in the $\{110\}\langle 110\rangle$ family can be paired with another, by permuting $\mathbf{n}_{(k)}^{(r)}$ and $\mathbf{m}_{(k)}^{(r)}$, where the slip rate is equal and the plastic spin is exactly opposite. As a result, the contribution to texture evolution due to plastic spin is limited to slip on the B systems essentially, since the computed activity on the $\mathrm{C}$ systems has been found very small.

Fig. 3 shows the texture history of halite in axial extension as predicted by the Taylor, VSC, SOE and TGT models, when the 45 initially uniformly-distributed orientations shown in Fig. 1a are used. In contrast with the similitude of the macroscopic stressstrain curves, the models now distinguish themselves by giving different patterns for the texture development. In the Taylor model, all poles move towards the [111] direction, with those initially in the center region of the triangle moving the fastest, and those near the lower [001]-[110] edge the slowest. The VSC model predicts almost the same pattern of texture evolution, except for some poles near the [001] corner that are heading to [001] slowly. The differences in the texture development can be interpreted, at least partly, in terms of the relative activities that these models predict (e.g., Wenk, 1999). Obviously, the dominant B slip and small amount of C slip for the Taylor model lead to a strong [111] texture. For the VSC model, the $\mathrm{B}$ systems are relatively less active, and some weak [001] texture is predicted. Similarly, the tangent model gives the least amount of $\mathrm{B}$ slip and produces a 
(a)

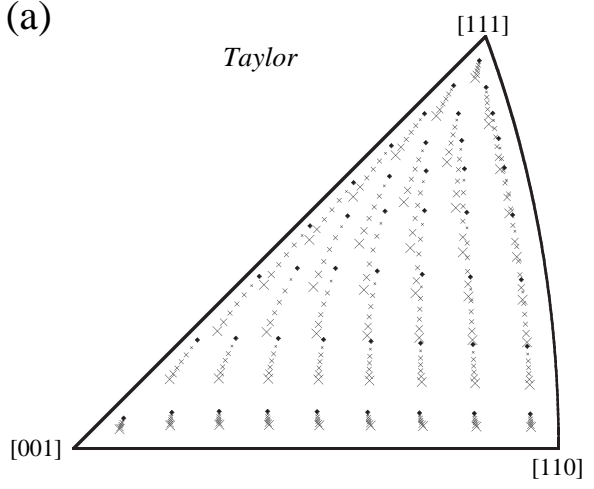

(c)

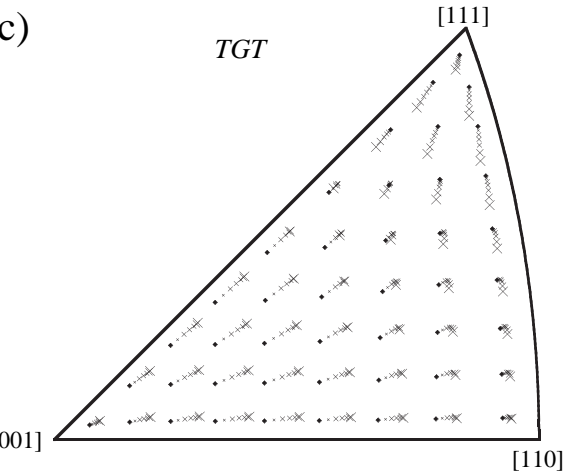

(b)

[001]

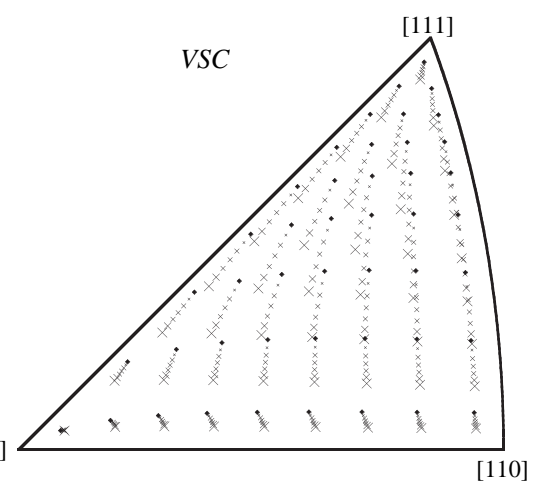

(d)

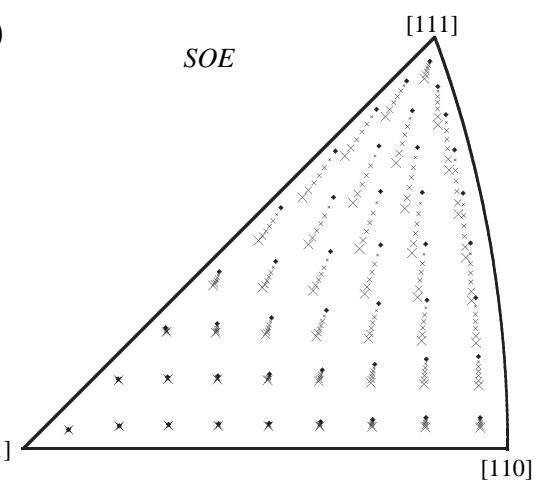

Fig. 3. Texture development of halite in axial extension at room temperature, according to the (a) Taylor model, the (b) variational (VSC), (c) tangent (TGT) and (d) second-order (SOE) self-consistent models. The largest cross symbols denote the initial poles and the diamond symbols denote their final positions after $30 \%$ axial strain. Smaller cross symbols correspond to $5 \%$ strain increments.

bimodal texture: about $1 / 4$ of the poles shift to [111] and give a peak with moderate density; the rest of the poles move towards [001] and form the main peak. The texture predicted by the second-order method develops a pattern that combines some characteristics of the Taylor and TGT estimates, i.e. the number of poles that move towards the [111] direction is much less than that in the Taylor estimate, but it is larger than with the TGT prediction. Other poles, especially those initially near the [001] corner, remain almost static during deformation. The resulting texture should thus be also bimodal, like the TGT prediction, but the primary peak should now be on the [111] corner, with a density of poles near the [001] almost unchanged from its initial value, and thus smoother than the TGT prediction.

The patterns shown in Fig. 3 are a perfect illustration of the large differences that are produced when a given homogenization model for linear materials, namely the self-consistent model, is extended by different methods (TGT, VSC, SOE) to nonlinear behaviors. Moreover, they also show that the global activity on a family of slip systems, the B-type in the present case, is not sufficient for interpreting the texture evolution.

\subsection{Texture predicted by the SOE method}

As has been mentioned previously, a set of 45 representative orientations reaches the limit of the computational power that was available for the second-order simulations. On the other hand, the set of 45 discrete orientations is inadequate for plotting contour lines. Therefore, 20 sets of 45 randomly distributed orientations were generated in the stereographic triangle, and were utilized individually for 
simulations with the second-order method. When combined with the result given by the set of 45 uniformly distributed orientations, this leads to a total of 945 final orientations that are used to produce a single pole figure where contour lines can be plotted. In concept, such a treatment is similar to the ensemble averaging that has been applied in the FFT simulations of Lebensohn et al. (2004a). However, since 21 independent simulations have been performed, the interactions between grains in different sets have been ignored, and the resulting deformation texture is not expected to be very accurate. Fig. 2a shows also the highest and lowest stress-strain relations that were obtained from the SOE simulations
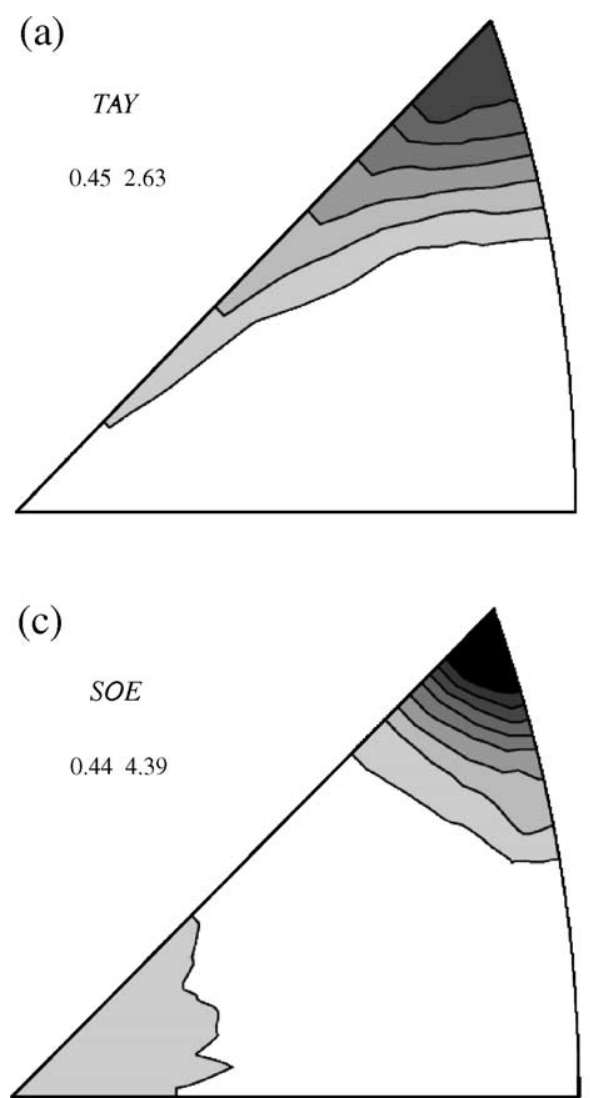

when using the 20 sets of 45 random orientation. The stress averaged over the $20 \mathrm{SOE}$ simulations is slightly higher than the SOE curve that uses 45 uniform orientations, by a maximum of $2.5 \mathrm{MPa}$ for a strain around 5\%. It can be observed that the highest SOE result almost overlaps with the HEP stress-strain curve, while the lowest one is still significantly higher than the TGT prediction.

It is nevertheless interesting to compare this ensemble-averaged result for the second-order model with other simulations, as in Fig. 4, where the 945 orientations were considered simultaneously for the Taylor and TGT models, and 4096 were used in the HEP simulations. Fig. $4 d$ uses data provided by R.A. Leben-

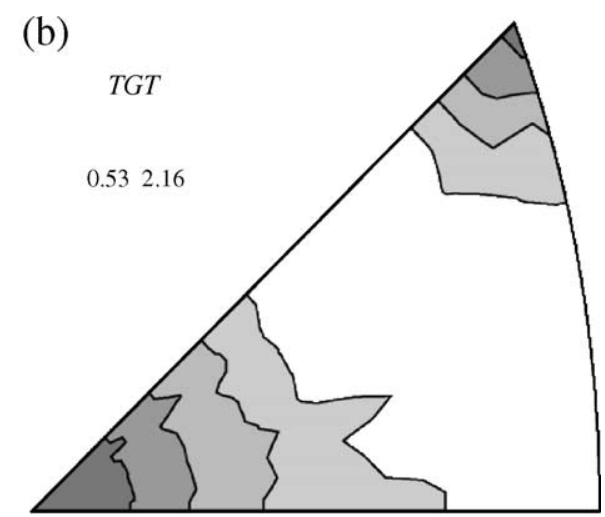

(d)
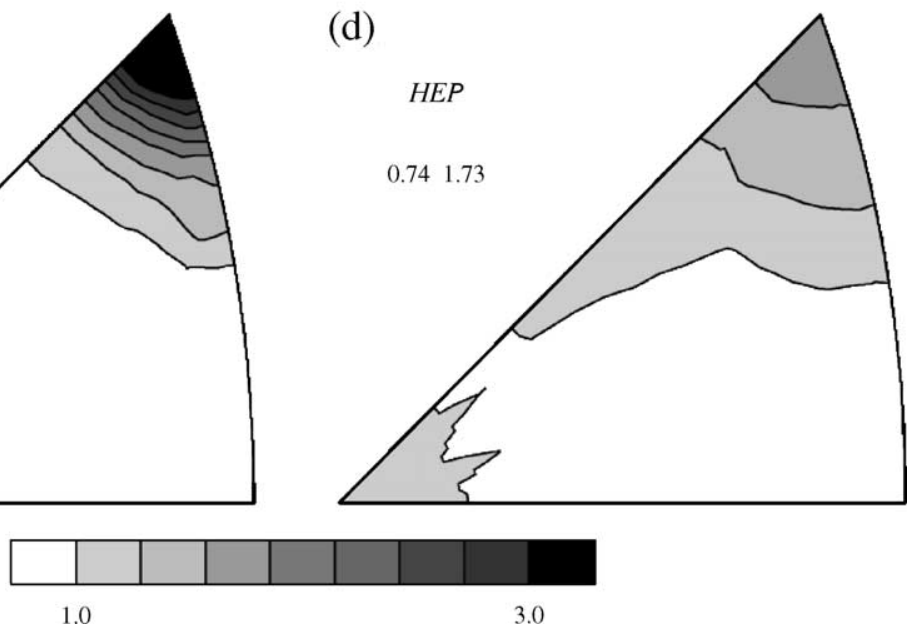

3.0

Fig. 4. Deformation texture of halite after 30\% axial strain at room temperature, according to (a) the Taylor model, (b) the tangent (TGT) and (c) second-order (SOE) self-consistent models, and (d) the "hybrid element polycrystal" (HEP) model (replotted from original data provided by R.A. Lebensohn). All figures use the same gray scale, and extremal values reached on each pole figure are also indicated. 
sohn, so that the same plotting program could be employed in order to reduce possible biases associated with the plotting routines. The predictions of the VSC method are of limited interest because of their similitude with the Taylor results, and are not shown. As already discussed in the previous subsection, the texture given by the SOE method is bimodal, but the intensity at [111] is found higher than with all other models. Even the Taylor model leads to a slightly lower value, probably because of the shoulder that forms along the [001]-[111] edge. The [001] area has an intensity that is comparable to what the HEP model gives, but the latter induces the lowest [111] peak among all models. This is again a distinguishing feature of the second-order estimates, as was the slope of the stress-strain curve and the level of the activity of the type-A systems, and it is important to test this result against the experimental measurements. In this connection, it is relevant to mention that Lebensohn et al. (2003) found that the peak intensities predicted by the TGT and HEP models were lower than the experimental observations, since the opposite is generally found when models are compared to experiments with other materials. Fig. 5 shows the pole figure obtained experimentally by Lebensohn et al. (2003) for a 15\% axial strain in extension, and the corresponding results given by the second-order method (combining 21 independent calculations, as explained above). (In this figure, the SOE predictions are shown in part $b$ using the same gray scale as in the experiments, and in part c using the same gray scale as in Fig. 4.) It should first be noted that the SOE texture shown in Fig. $5 \mathrm{c}$ is less sharp than that shown in Fig. 4c, with the same contour levels, which means that the texture continues
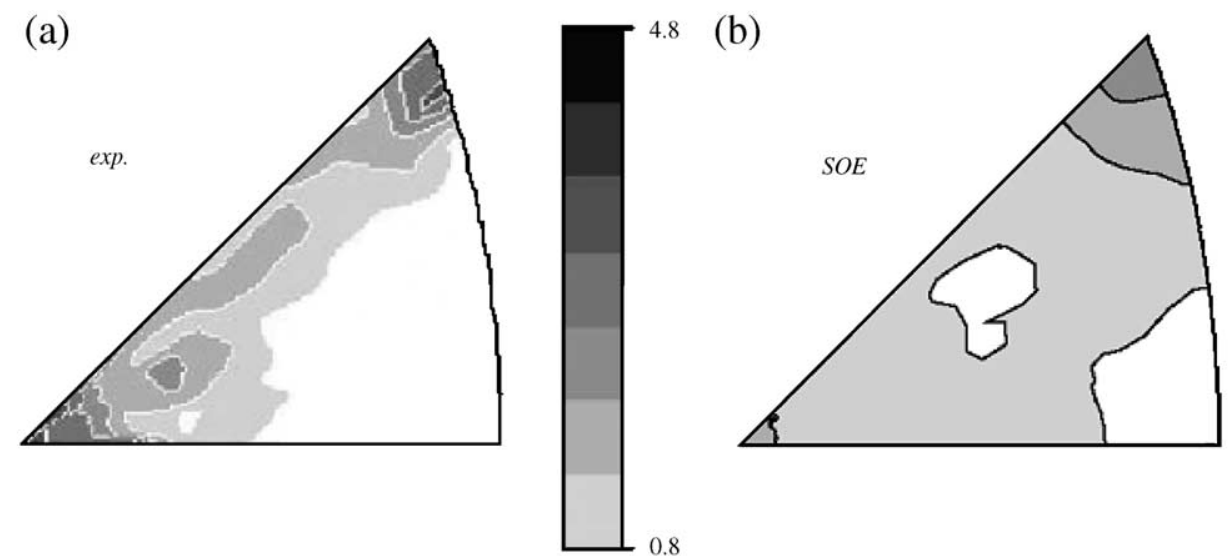

0.8
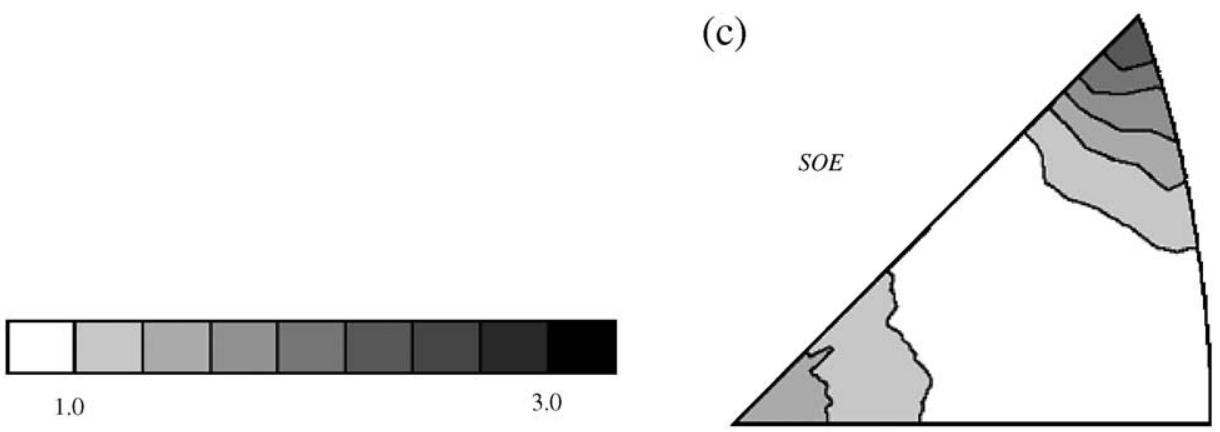

Fig. 5. Deformation texture of halite after $15 \%$ axial strain at room temperature. Comparison between (a) the experimental measures taken from Lebensohn et al. (2003) and the predictions of the second-order method. The latter results are plotted using two different gray scales: (b) the same scale as the experiments, or (c) the same scale of in Fig. 4. 
to evolve significantly between $15 \%$ and $30 \%$ axial strain. Moreover, the measured [111] peak is higher than the simulation (compare Fig. 4a and b, with the same contour levels), and thus the predicted [111] peak does not seem excessively large, as might have been erroneously deduced when the SOE texture was found sharper than the Taylor one in Fig. 4. The [001] peak is even slightly lower than the experimental measure, but still higher than with the Taylor, TGT, and HEP results (second line of Fig. 14 of Lebensohn et al., 2003). In summary, the SOE prediction shows the best agreement with the experimental pole figure at $15 \%$ axial strain, amongst all models.

\section{Concluding remarks}

In this work, the second-order self-consistent method has been used, for the first time, to simulate texture evolution in viscoplastic polycrystals. Halite at room temperature has been chosen as the relevant material system because of its strongly nonlinear and anisotropic constitutive behavior. It should be mentioned in this context that a study of halite at high-temperature conditions was carried out by Gilormini et al. (2003) making use of the earlier "variational" method. At high temperature the halite crystals exhibit fairly isotropic behavior, leading to nearly uniform fields in the polycrystal, and so the Taylor model is quite sufficient for predicting the effective behavior and texture evolution in halite at such temperatures. On the other hand, at room temperature, where the fields in the polycrystal are strongly heterogeneous, the differences between the Taylor and various self-consistent models are much more significant. In this study, it has been found that the overall performance of the "second-order" method, as measured by comparisons with the corresponding "hybrid element polycrystal" simulations, appears to be better than the Taylor, variational self-consistent and tangent self-consistent models. It also gives patterns for the stress-strain relation and texture development that are more consistent with the experimental observations. It is conjectured that the texture development patterns predicted by these models provide more reliable measures of overall accuracy than the macroscopic stress-strain relations. This is because the latter can be more easily adjusted by choosing the crystal parameters within physically meaningful ranges, at least for a specific type of loading condition, while the former are less sensitive to the crystal parameters, and more sensitive to the particular model used in the simulation.

In all the simulations of texture evolution carried out in this work, as well as in most numerical works found in the literature, the basic hypothesis is made that all crystals with the same initial orientation rotate as one unit, or phase, whose movement is assumed to be controlled by the "average" quantities over all grains in that phase. Consequently, the orientations of the grains in any phase remain the same throughout the evolution process. However, recent work (Lebensohn et al., 2004a,b) has shown that the strain-rate (and stress) fields inside the grains can exhibit significant fluctuations, especially for highly nonlinear and anisotropic constituent grains, which suggests that the above evolution hypothesis may be too simplistic. In fact, this hypothesis is similar, in some sense, to the "oneelement-per-orientation" scheme employed in standard FEM simulations of polycrystals (e.g., Balasubramanian and Anand, 2002), which should be less accurate than more sophisticated numerical methods, such as the FFT full-field simulation (e.g., Michel et al., 2000; Lebensohn, 2001). Moreover, given that the new second-order self-consistent method also yields estimates for the fluctuations of the strainrate field in the grains (Liu and Ponte Castañeda, 2004a,b), it is natural to suggest that improved schemes for the texture evolution in polycrystals may be possible making use of the additional fluctuation information. Of course, such improved schemes would require a more complete kinematical description of the texture development process, which is yet to be elucidated.

\section{Acknowledgments}

This work was supported in part by the NSF under Grant No. CMS-0201454 and in part by an NSFCNRS international collaborative project (NSF Grant No. OISE-0231867). The HEP results used as references were kindly provided by R.A. Lebensohn 
(LANL, USA). C.N. Tomé (LANL, USA) is gratefully acknowledged for providing his program for plotting pole figures. Valuable suggestions by an anonymous referee are also gratefully acknowledged.

\section{References}

Adams, B.L., Olson, T., 1998. The mesostructure-properties linkage in polycrystals. Prog. Mater. Sci. 43, 1-88.

Aubertin, M., Hardy Jr., H.R., 1998. The mechanical behavior of salt. Proceedings of the Fourth Conference. Series on Rock and Soil Mechanics, vol. 22, p. 658.

Balasubramanian, S., Anand, L., 2002. Plasticity of initially textured hexagonal polycrystals at high homologous temperatures: application to titanium. Acta Mater. 50, 133-148.

Barton, N.R., Dawson, P.R., 2001. On the spatial variations in orientations and misorientations in two-phase titanium. Mod. Sim. Mat. Sci. 9, 433-463.

Beaudoin, A.J., Dawson, P.R., Mathur, K.K., Kocks, U.F., 1995. A hybrid finite element formulation for polycrystal plasticity with consideration of macrostructural and microstructural linking. Int. J. Plast. 11, 501-521.

deBotton, G., Ponte Castañeda, P., 1995. Variational estimates for the creep behaviour of polycrystals. Proc. R. Soc. Lond., A 448, $121-142$.

Bunge, H.J., Esling, C. (Eds.), 1982. Quantitative texture analysis, Deutsche Gesellschaft für Metallkunde.

Carter, N.L., Heard, H.C., 1970. Temperature and rate dependent deformation of halite. Am. J. Sci. 269, 193-249.

Carter, N.L., Hansen, F.D., 1983. Creep of rocksalt. Tectonophysics 92, 275-333.

Carter, N.L., Horseman, S.T., Russel, J.E., Handin, J., 1993. Rheology of rocksalt. J. Struct. Geol. 15, 1257-1271.

Franssen, R.C.M.W., 1994. The rheology of synthetic rocksalt in uniaxial compression. Tectonophysics 233, 1-40.

Gilormini, P., Liu, Y., Ponte Castañeda, P., 2003. Homogenizationbased predictions for texture evolution in halite. In: Miehe, C. (Ed.), Computational Mechanics of Solid Materials at Large Strains (IUTAM Symposium). Kluwer Academic Publishers, New York, pp. 269-278.

Guillopé, M., Poirier, J.P., 1979. Dynamic recrystallization during creep of single-crystalline halite: an experimental study. J. Geophys. Res. 84, 5557-5567

Heard, H.C., 1972. Steady-state flow in polycrystalline halite at pressure of 2 kilobars. In: Heard, H.C., Borg, I.Y., Carter, N.L., Raleigh, C.B. (Eds.), Flow and Fracture of Rocks, Geophysical Monograph. American Geophysical Union, pp. 191-210.

Hill, R., 1965. Continuum micro-mechanics of elastoplastic polycrystals. J. Mech. Phys. Solids 13, 89-101.

Hunsche, U., Hampel, A., 1999. Rock salt-the mechanical properties of the host rock material for a radioactive waste repository. Eng. Geol. 52, 271-291.

Hutchinson, J.W., 1976. Bounds and self-consistent estimates for creep of polycrystalline materials. Proc. R. Soc. Lond., A 348, $101-127$.
Kern, H.M., 1979. Texture development in calcite and quartz deformation at uniaxial and real triaxial states of strain. Bull. Mineral. 102, 290-300.

Kocks, U.F., Tomé, C.N., Wenk, H.-R., 1998. Texture and Anisotropy. Cambridge University Press.

Lebensohn, R.A., 2001. $N$-site modelling of a 3D viscoplastic polycrystal using the Fast Fourier Transform. Acta. Mater. 49, $2723-2737$.

Lebensohn, R.A., Tomé, C.N., 1993. A self-consistent anisotropic approach for the simulation of plastic deformation and texture development of polycrystals: application to zirconium alloys. Acta Metall. Mater. 41, 2611-2624.

Lebensohn, R.A., Tomé, C.N., 1994. A self-consistent viscoplastic model: calculation of rolling textures of anisotropic materials. Mater. Sci. Eng., A 175, 71-82.

Lebensohn, R.A., Dawson, P.R., Kern, H.M., Wenk, H.R., 2003. Heterogeneous deformation and texture development in halite polycrystals: comparison of different modeling approaches and experimental data. Tectonophysics 370, 287-311.

Lebensohn, R.A., Liu, Y., Ponte Castañeda, P., 2004a. Macroscopic properties and field fluctuations in model power-law polycrystals: full-field solutions versus self-consistent estimates. Proc. R. Soc. Lond., A 460, 1381-1405.

Lebensohn, R.A., Liu, Y., Ponte Castañeda, P., 2004b. On the accuracy of the self-consistent approximation for polycrystals: comparison with full-field numerical simulations. Acta Mater. $52,5347-5361$.

Lipinski, P., Krier, J., Berveiller, M., 1990. Elastoplasticité des métaux en grandes déformations: comportement global et évolution de la structure interne. Rev. Phys. Appl. 25, 361-388.

Liu, Y., 2003. Macroscopic behavior, field fluctuations and texture evolution in viscoplastic polycrystals. Ph.D. Thesis, University of Pennsylvania, U.S.A.

Liu, Y., Ponte Castañeda, P., 2004a. Second-order estimates for the effective behavior and field fluctuations in viscoplastic polycrystals. J. Mech. Phys. Solids 52, 467-495.

Liu, Y., Ponte Castañeda, P., 2004b. Homogenization estimates for the average behavior and field fluctuations in cubic and hexagonal viscoplastic polycrystals. J. Mech. Phys. Solids 52, 1175-1211.

Liu, Y., Gilormini, P., Ponte Castañeda, P., 2003. Variational selfconsistent estimates for texture evolution in viscoplastic polycrystals. Acta Mater. 51, 5425-5437.

Mandel, J., 1972. Plasticité et viscoplasticité. CISM Udine Courses and Lectures, vol. 97. Springer-Verlag, New-York.

Molinari, A., Ganova, G.R., Ahzi, S., 1987. A self-consistent approach of the large deformation polycrystal plasticity. Acta Metall. Mater. 35, 2983-2994.

Michel, J., Moulinec, H., Suquet, P., 2000. A computational method based on augmented Lagrangians and fast Fourier transforms for composites with high contrast. Comput. Model. Eng. Sci. 1, 79-88.

Nebozhyn, M.V., 2000. Variational self-consistent estimates for the effective behavior of viscoplastic polycrystals. Ph.D. Thesis, University of Pennsylvania, U.S.A.

Nebozhyn, M.V., Gilormini, P., Ponte Castañeda, P., 2001. Variational self-consistent estimates for cubic viscoplastic polycrys- 
tals: the effect of grain anisotropy and shape. J. Mech. Phys. Solids 49, 313-340.

Ogden, R.W., 1984. Non-linear Elastic Deformations. Ellis Harwood, Chichester, p. 130.

Peach, C.J., Spiers, C.J., 1996. Influence of crystal plastic deformation on dilatancy and permeability development in synthetic salt rock. Tectonophysics 256, 101-128.

Ponte Castañeda, P., 1997. Nonlinear composite materials: effective constitutive behavior and microstructure evolution. In: Suquet, P. (Ed.), Continuum Micromechanics, CISM Courses and Lecture Notes, vol. 377. Springer-Verlag, Wien, pp. 131-195.

Ponte Castañeda, P., 2002. Second-order homogenization estimates for nonlinear composites incorporating field fluctuations. ITheory. J. Mech. Phys. Solids 50, 737-757.

Ponte Castañeda, P., Suquet, P., 1998. Nonlinear composites. Adv. Appl. Mech. 34, 171-202.

Reuss, A., 1929. Calculation of the flow limits of mixed crystals on the basis of the plasticity of the monocrystals. Z. Angew. Math. Mech. 9, 49-58.

Siemes, H., 1974. Anwendung der Taylor Theorie auf die Regelung von Kubischenmineralen. Contrib. Mineral. Petrol. 43, $149-157$.
Taylor, G.I., 1938. Plastic strains in metals. J. Inst. Met. 62, 307-324.

Ter Heege, J.H., De Bresser, J.H.P., Spiers, C.J., in press. Rheological behaviour of synthetic rocksalt: the interplay between water, dynamic recrystallization and deformation mechanisms. Journal of Structural Geology.

Watanabe, T., Peach, C.J., 2002. Electrical impedance measurement of plastically deforming halite rocks at 125 degrees $\mathrm{C}$ and 50 MPa. J. Geophys. Res. Solid Earth B1 (107) (Art. No. 2004).

Wawersik, W.R., Zeuch, D.H., 1986. Modelling and mechanistic interpretation of creep of rock salt below $200^{\circ}$. Tectonophysics $121,125-152$.

Wenk, H.R., 1999. A voyage through the deformed Earth with the self-consistent model. Model. Simul. Mater. Sci. Eng. 7, $699-722$.

Wenk, H.R., Ganova, G., Molinari, A., Mecking, H., 1989. Texture development in halite: comparison of Taylor model and selfconsistent theory. Acta Metall. 37, 2017-2029.

Willis, J.R., 1977. Bounds and self-consistent estimates for the overall moduli of anisotropic composites. J. Mech. Phys. Solids $25,185-202$. 